\title{
Estadísticas de salud en el Perú: mejor llenado de información y su apropiada utilización para promover la salud
}

\section{Health statistics in Peru: improving the quality of data collection and its proper use for promoting health (care)}

Correspondencia

Alberto Zolezzi

actamedicaperuana@cmp.org.pe

Recibido: $13 / 12 / 2017$

Aprobado: 15/12/2017

Citar como: Zolezzi A.

Estadísticas de salud en el Perú: mejor llenado de información y su apropiada utilización para promover la salud. Acta Med Peru. 2017;34(4): 257-8

\section{Alberto Zolezzi ${ }^{1}$}

1 Director Acta Médica Peruana

Los datos estadísticos que describen el estado de la salud en el Perú son importantes para determinar las tareas a seguir en salud y decidir las prioridades que debemos considerar en el enfoque a la atención de la salud. El Ministerio de Salud (MINSA) peruano busca contar con la mayor y mejor cantidad de información sanitaria que le permita tomar mejores decisiones. Actualmente, esto se logra con la recopilación obligatoria de información que se realiza en los establecimientos de salud: nacimientos, inmunizaciones, reportes de enfermedades comunicables y datos de las defunciones que se obtienen en el certificado respectivo.

El Perú actualmente tiene aproximadamente una población de 31 millones de habitantes, de los que el $29 \%$ corresponde a menores de 15 años y el $9 \%$ a mayores de 60 años. La mayoría de su población (78\%) vive en zonas urbanas. Para el año 2012, la esperanza de vida al nacer fue de 77 años y la esperanza de vida a los 60 años, es decir, el número de años en promedio que esperaría vivir una persona de 60 años si las condiciones de mortalidad no variaran durante el resto de su vida, fue de 23 años; además, una esperanza de vida saludable de 67 años (Tabla 1). La esperanza de vida durante el periodo 2000-2012 ha aumentado en cinco años, pero hay 10 años de vida con molestias crónicas que van a deteriorar la calidad de vida.

Los datos oficiales presentados en el informe de la Organización Mundial de la Salud respecto al Perú ${ }^{[1]}$ indica (Figura 1) el poco dinero invertido en salud, que corresponde al $10-20 \%$ del utilizado entre otros países de la región: un gasto per cápita de 300-600 dólares en promedio frente a un gasto que supera los 3000 dólares.

Los datos de mortalidad son más útiles gracias a un mejor llenado de los certificados de defunción, aunque aún el $30 \%$ son completados en forma inapropiada. Los resultados indican que las infecciones respiratorias, los problemas cardiovasculares isquémicos, la cirrosis y los accidentes automovilísticos son los máximos responsables de la mortalidad.

Entonces, el paso adelante que tenemos que dar es trabajar en minimizar los efectos de una calidad de vida disminuida y la perdida de años de vida potencialmente útil.

Los datos de morbilidad son un aspecto que requiere un mejor desarrollo. Las estadísticas oficiales informan de las enfermedades por grupos (respiratorias, de la piel, neurológicas, etc.) ${ }^{[2]}$, sin dar datos de diagnósticos más detallados, por enfermedades, haciendo que muchas veces se defina la prevalencia de las enfermedades en relación a muestreos pequeños y/o de lugares (hospitales o áreas de salud calculan la prevalencia de ciertas enfermedades) que no reflejan a todo el país. 
Tabla 1. Esperanza de vida (años), 2012.

\begin{tabular}{ccccc}
\hline \multicolumn{5}{c}{ Esperanza de vida (años), 2012} \\
\hline $\begin{array}{c}\text { Perú } \\
\text { Rida }\end{array}$ & $\begin{array}{c}\text { Región } \\
\text { OMS }\end{array}$ & $\begin{array}{c}\text { Grupo } \\
\text { ingresos } \\
\text { BM }\end{array}$ \\
\hline $\begin{array}{c}\text { Esperanza de } \\
\text { vidacer }\end{array}$ & A los 60 años & 23 & 78 & 74 \\
$\begin{array}{c}\text { Esperanza de } \\
\text { vida saludable }\end{array}$ & Al nacer & 67 & 67 & 66 \\
\hline
\end{tabular}

El desarrollo de estudios nacionales orientados a calcular la morbilidad es una tarea pendiente. Desde hace más de 20 años se ha implementado el llenado de diagnósticos según sistema de información de salud (HIS). El HIS, que tiene como característica de la atención médica la posibilidad de incluir hasta tres diagnósticos, es una herramienta que si bien puede ser inadecuada para estudios clínicos precisos, si es monitoreada y seguida en su uso, puede ser útil para el estudio de prevalencia e incidencia de enfermedades ${ }^{[3]}$.

Los estudios nacionales de morbilidad en establecimientos del MINSA y Gobiernos Regionales describen que las patologías más frecuentes son las infecciones respiratorias superiores ( $16 \%$ de los diagnósticos de consulta externa), seguida por patología bucal (15\%), Infecciones intestinales $(3,4 \%)$, patología gástrica y duodenal $(3,2 \%)$, otras molestias urológicas $(3,2 \%)$ y las dorsopatías $(3,2 \%)$. Cabe resaltar la información clínica brindada por hospitales como Arzobispo Loayza del Centro de Lima, Cayetano Heredia de San Martín de Porres y María Auxiliadora de San Juan de Miraflores ${ }^{[4-6]}$, registrada ya por varios años

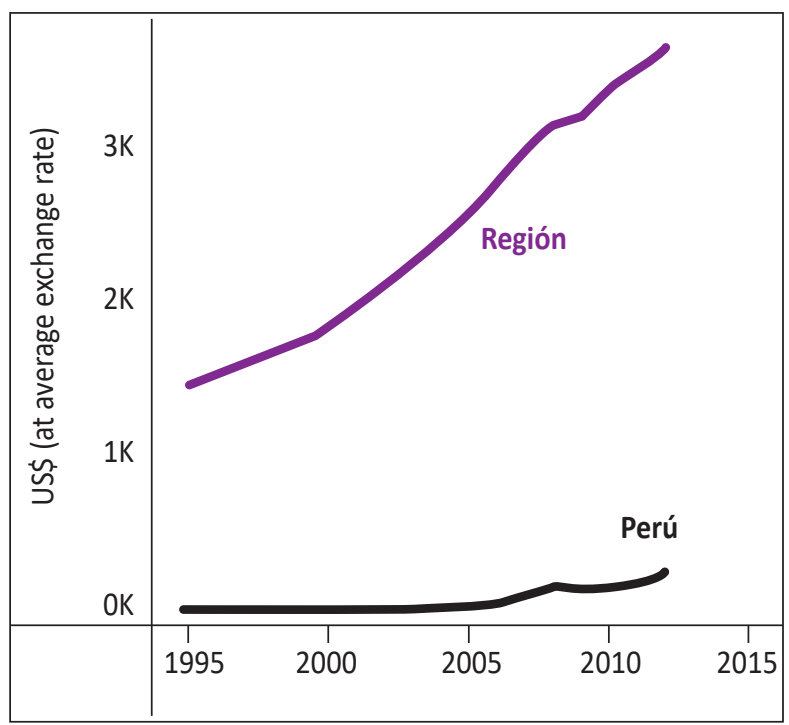

Figura 1. Gastos totales per cápita empleados en salud. seguidos en un anuario estadístico que es de utilidad para tener una idea de las enfermedades más frecuentes diagnosticadas en la consulta externa, y con ello hacer una comparación con los resultados nacionales.

Lo que salta a la vista es que el diagnóstico más común en los tres hospitales es la hipertensión arterial, seguido por dorsalgias, dispepsia, trastornos de la refracción, rinofaringitis, litiasis vesicular, hiperplasia de próstata y diabetes. Aquí notamos que los diagnósticos por "daños" a nivel nacional pueden omitir diagnósticos que pueden requerir un medio diagnóstico mínimo como es la toma de la presión o la medición de la vista. De igual manera, los hospitales dejan los consultorios de salud oral con un espacio menor, mientras que en los centros primarios de atención, junto a las cuatro grandes especialidades (medicina, pediatría, ginecoobstetricia y cirugía), el consultorio de salud oral es un baluarte de la atención comunitaria.

Así, hay que entender a la salud como un concepto de bienestar completo, y esto es lo que puede diferenciar cómo entre los tres hospitales con información de morbilidad, el diagnóstico de obesidad es el segundo más frecuente en el Hospital Cayetano Heredia, quedando en el $18^{\circ}$ y $30^{\circ}$ diagnósticos en los hospitales María Auxiliadora y Loayza respectivamente; lo que reflejaría un compromiso por diagnosticar este problema nutricional en dicho hospital. Aspectos tan sencillos como el pesar a los pacientes puede ser omitido en hospitales con gran carga asistencial.

Finalmente, considerando que hay datos en los que la pérdida prematura de la vida o una incapacidad para tener una vida saludable pueden restar hasta miles de años de una vida plena para los peruanos, es de vital importancia que se mejoren la calidad de los procesos del HIS, lo cual permitirá contar con información real y oportuna que facilite la toma de decisiones dentro del sistema de salud peruano.

\section{REFERENCIAS BIBLIOGRÁFICAS}

1. World Health Organization. Global Health Observatory (GHO) data. Geneva: WHO; c2017 [citado el 7 de noviembre de 2017]. Disponible en: http://www.who.int/gho/en/

2. Ministerio de Salud del Perú, Dirección General de Epidemiología. Análisis de situación de salud del Perú. Lima: Minsa; 2013.

3. Ward MM. Estimating disease prevalence and incidence using administrative data: some assembly required. J Rheumatol. 2013;40(8):1241-3.

4. Hospital María Auxiliadora, Oficina de Estadística e Informática. Compendio Estadístico 2016. Lima: Hospital María Auxiliadora; 2016.

5. Hospital Nacional Cayetano Heredia. Análisis Situacional de Salud 2015. Lima: Hospital Nacional Cayetano Heredia; 2015.

6. Hospital Nacional Arzobispo Loayza, Oficina de Epidemiología y Salud Ambiental. Análisis de la Situación de Salud 2008. Lima: Hospital Nacional Arzobispo Loayza; 2009. 